\title{
El rol del lenguaje natural en la cognición: un análisis del neowhorfismo
}

\section{The Role of Natural Language in Cognition: an Analysis of Neowhorfianism}

\author{
MARINA ORTEGA-ANDRÉS \\ Universidad del País Vasco
}

Recibido: 08/03/2016 Aceptado:06/09/2016

\begin{abstract}
RESUMEN
La tesis de que los hablantes de distintas lenguas presentan diferencias cognitivas ha sido defendida por lingüistas, psicólogos y filósofos. Muchos resultados dan a entender que existe una correlación entre lenguaje y pensamiento, pero no hay una explicación teórica precisa sobre cómo influye el lenguaje a la cognición. El objetivo de estas páginas es estudiar qué mecanismos son más explicativos de los resultados empíricos. Para ello, se analizan algunos estudios empíricos y se extraen cinco requisitos que la explicación teórica del fenómeno debe cumplir. Se analizan cinco explicaciones y se argumenta a favor de la tesis de la re-representación.
\end{abstract}

\section{PALABRAS CLAVE \\ RELATIVIDAD LINGÜÍSTICA, LENGUAJE NATURAL, CATEGORÍAS MENTALES, COGNICIÓN}

\begin{abstract}
The thesis that speakers of different languages show cognitive differences has been supported

* Esta investigación ha sido posible gracias a la financiación recibida por el grupo de investigación de MINECO Lexical Meaning and Concepts (FFI2014-52196-P), Research Group for Theoretical Linguistics (hiTT Ref. IT769-1) y la Beca de iniciación de la investigación para estudiantes de máster 2015 del Vicerrectorado de Investigación de la UGR. Asimismo, quiero expresar mi agradecimiento a Agustín Vicente y a Juan José Acero.
\end{abstract}

(C) Contrastes. Revista Internacional de Filosofía, vol. XXII-N ${ }^{\circ} 1$ (2017), pp. 173-190. ISSN: 1136-4076

Departamento de Filosofía, Universidad de Málaga, Facultad de Filosofía y Letras Campus de Teatinos, E-29071 Málaga (España) 
by linguistics, psychologist and philosophers. Many results suggest that there is a correlation between language and thought, but there is not a precise theoretical explanation of how language influences cognition. The aim of this paper is to analyze which explanatory mechanisms give an account of the results. First, I will analyze some empirical studies. Second, I will sketch five possible theoretical explanations. I argue for the theory of re-representation.

KEYWORDS

LINGUISTIC RELATIVISM, NATURAL LANGUAGE, MENTAL CATEGORIES, COGNITION

\section{INTRODUCCIÓN}

SEGÚN LA TESIS DE LA RELATIVIDAD LINGÜÍSTICA (TRL) propuesta por Edward Sapir (1929) y Benjamin Lee Whorf (1956/2012), la manera en que piensan los individuos depende de la lengua que hablen normalmente. Actualmente se sostiene que el lenguaje natural (LN) influye en la formación de conceptos. Los defensores de esta tesis tienen que lidiar con la gran diversidad de opiniones y subtesis que hay en torno a ella. Esta diversidad impide que haya un veredicto final sobre la influencia de la lengua en la estructura conceptual (Vicente y Martínez-Marinque 2013).

En este trabajo me concentro en la influencia de la lengua en algunos dominios de pensamiento abstracto y relacional. Para defender la TRL es necesario aportar una explicación teórica sobre cómo el LN influye en la cognición. Sin esta explicación, la tesis neowhorfista está incompleta. El objetivo de estas páginas es estudiar algunos mecanismos teóricos y analizar la capacidad explicativa que tiene cada uno de ellos para dar cuenta de los datos empíricos. Se analizan cinco perspectivas teóricas, de las cuales argumentaré que la más apropiada es la teoría de la Re-representación. Para ello, los primeros apartados están dedicados a analizar algunos estudios empíricos y estudiar qué conclusiones teóricas se extraen de ellos. En el último apartado se extraen las exigencias que los estudios imponen y se proporcionan cinco mecanismos teóricos por los que el LN puede influir en algunos dominios cognitivos. Las propuestas son las que siguen: la hipótesis del LN como herramienta (LH); la hipótesis del mapeo asociativo intermodular (MA); la hipótesis de los conceptos léxicos y modelos cognitivos (LCCM); la hipótesis del etiquetaje lingüístico (EL) y la teoría de la redescripción de representaciones (RR). Por último, se estudia cuáles de ellas resuelven los requisitos extraídos de los resuiltados empíricos.

\section{Pensamiento abSTRacto: El TIEMPo}

Resulta natural pensar que la influencia del LN en la cognición debe 
ser más explícita en dominios donde la percepción ejerza menor influencia. Siendo así, podríamos distinguir entre dominios más imaginarios y dominios más lingüísticos. Boroditsky y Prinz (2008) defienden que el LN tiene un rol muy importante en la construcción de representaciones de entidades abstractas que no se pueden adquirir perceptivamente. Boroditsky (2001) estudia las diferencias en la categorización de fenómenos temporales entre los hablantes del chino mandarín y el inglés. Mientras que en inglés se utilizan metáforas horizontales para ordenar eventos temporalmente, en chino mandarín se utilizan metáforas tanto horizontales como verticales. El estudio muestra que los hablantes nativos del inglés y el chino mandarín presentan diferencias al realizar tareas relacionadas con el dominio del tiempo. Los hablantes del inglés verificaron más rápidamente la oración «Marzo es previo a Abril» después de observar imágenes en colocación horizontal. En cambio, los hablantes del mandarín necesitaron menos tiempo después de haber sido expuestos a estímulos verticales que tras los estímulos horizontales. La conclusión que extrae Boroditsky es que el LN tiene un rol importante en la modelación del pensamiento en dominios abstractos y relacionales. Esta hipótesis ha sido defendida y analizada posteriormente muchas veces (Acero 2013; January y Kako 2007; Nuñez y Sweester 2006; Casasanto y Boroditsky 2007; Chen 2007; Casasanto 2007; Casasanto 2015).

Casasanto y Boroditsky (2007) explican que cuando hablamos del tiempo solemos utilizar metáforas de dominios más concretos o perceptualmente más ricos. Lo interesante de estos casos son las diferencias interlingüísticas que existen entre la conducta de los hablantes que utilizan unas u otras metáforas. Los estudios muestran que no en todas las lenguas se utilizan metáforas espaciales, sino que en otras, como el griego y el castellano, se utilizan metáforas de cantidad. De estos estudios se extrae que hay una relación entre el uso de estas metáforas y la cognición (véase más abajo).

La mayoría de los resultados muestran que existe una correlación entre el LN y la conducta no lingüística, pero no que la relación sea causal. No basta con determinar que hay una coincidencia en la variación de comportamiento y el uso lingüístico, sino que hay que estudiar si esas diferencias interlingüísticas se deben al LN o a otros factores ambientales. El experimento de Casasanto (2007) intenta resolver esta cuestión estudiando las diferencias conductuales entre los hablantes nativos del griego y del inglés. Los hablantes del inglés utilizan metáforas espaciales para hablar del tiempo, mientras que los del griego utilizan metáforas de volumen o cantidad. El estímulo consiste en líneas que se alargan hasta desaparecer y en contenedores que se llenan de agua. En la primera parte del experimento hay variación entre los hablantes de las dos lenguas al estimar la duración del estímulo, a pesar de que la duración es constante. Los hablantes del inglés tienden a estimar que las líneas que crecen 
menor distancia tardan menos en hacer el recorrido, mientras que los hablantes del griego muestran diferencias de estimación dependiendo de cuánto se llena un contenedor de líquido. El criterio de evaluación no es verbal, es decir, no se trata de que los hablantes describen el movimiento de los objetos de una u otra forma, sino que hay diferencias entre cuanto estiman que durará el evento.

En la segunda parte del experimento, Casasanto habitúa a los y las hablantes a utilizar metáforas de la otra lengua. Los hablantes del inglés fueron forzados a usar las metáforas de cantidad y los hablantes del griego a utilizar metáforas espaciales. Tras un proceso de habituación al uso de metáforas espaciales para hablar del tiempo, los resultados de los hablantes de griego se asemejaron a los resultados de hablantes del inglés. Los hablantes del inglés, por su parte, tras habituarse al uso de metáforas de cantidad para hablar del tiempo, dieron resultados similares a los hablantes del griego en la primera parte del experimento. La inversión de los resultados en la segunda fase muestra que es el uso de las metáforas lo que afecta a la estimación temporal y no otros factores sociales o culturales. La relación entre el LN y la cognición es causal.

Otra conclusión que se extrae es que los cambios que provoca la lengua en el pensamiento no son permanentes, sino que basta un periodo de uso a otras formas lingüísticas para que se transforme el pensamiento. Algunos autores (Landau, Dessalegn y Goldberg 2010) defienden que el proceso de transformación del pensamiento no puede ser algo que tenga lugar durante la adquisición de la lengua y cuyos cambios sean permanentes hasta la edad adulta, sino que las estructuras transformadas por el lenguaje deben ser lo suficientemente maleables como para que puedan volver a moldearse en un futuro. La explicación que se proponga sobre del rol del $\mathrm{LN}$ en el proceso de categorización tiene que permitir que los conceptos (o algunos de ellos) no sean modificados de forma permanente.

Aunque aquí sólo se hayan analizado estudios sobre cómo la lengua influye en cómo pensamos sobre el tiempo, otros campos de estudio, como el de los números (Casasanto 2005; Gordon 2004; Gelman y Gallistel 2004; Miller et al 2000; Pica et al. 2004; Spelke y Tsvkin 2001; Everett 2013) muestran que el tiempo no es el único ámbito abstracto en el que el LN está involucrado. De ahí que podamos decir que el LN influye en cómo pensamos en, al menos, algunos dominios abstractos.

\section{RELACIONES ESPACIALES}

En el apartado anterior se mostró evidencia empírica a favor de que el LN influye en al menos algunos ámbitos abstractos del pensamiento. Este apartado lo dedico a estudiar la influencia del $\mathrm{LN}$ en dominios relacionales, en 
concreto categorías relacionales del espacio.

Boroditsky y Gentner (2001) sostienen que tanto el dominio cognitivo como el lingüístico tienen un papel en la formación de conceptos, la influencia de un dominio u otro será mayor según los distintos tipos de palabras. Cuando predomina el dominio lingüístico, el mundo presenta información perceptiva que no está previamente organizada y que el LN categoriza. Cuando el dominio es de lo cognitivo-perceptual, los conceptos están ya organizados en categorías que son nombradas por el lenguaje. Su experimento apoya la idea de que los términos relacionales -como los verbos, las preposiciones, etc.- presentan mayores diferencias interlingüísticas que otras formas léxicas durante el desarrollo cognitivo. Aprender la denotación de estas palabras requiere un conocimiento previo del LN. Los objetos y las entidades se individúan prelingüísticamente, se aíslan los objetos perceptivamente. En cambio, cuando aprendemos una palabra que designa una relación, aislar la relación es sólo parte del trabajo, se tiene que descubrir qué combinación de los elementos conceptuales sirve como referente del término. De esta idea parte lo que ellas llaman la relatividad relacional, según la cual, los términos relacionales tienen un papel importante en la cognición y eso hace que haya diferencias en algunos ámbitos cognitivos entre los hablantes de lenguas que presentan diferencias léxicas importantes en estos términos.

Un ejemplo de pensamiento relacional es la colocación de objetos en el espacio que requiere utilizar sistemas de posición espacial. Los neowhorfistas han investigado cómo los sistemas de colocación espacial afectan a la conducta no lingüística (Pederston 1995; Brown y Levinson 2000; Li y Gleitman 2002; Levinson 2003; Majid et al. 2004). El estudio de Levinson (2003) parte de que hay tres sistemas de orientación espacial: el sistema absoluto -que utiliza los puntos cardinales Norte, Sur, Este y Oeste-, intrínseco - que toma como referencia la posición del hablante - y el relativo - que toma como referencia un segundo objeto. El estudio es interesante porque en él se investiga si la lengua influye en tareas no lingüísticas. Si el neowhorfismo defiende que el LN influye en los procesos psicológicos porque organiza el mundo en categorías que difieren de aquellas que descubriríamos sin el LN (Prinz y Reinees 2009), entonces tiene que demostrar que el LN afecta a la cognición en tareas no lingüísticas (Li, Duham y Carey 2009; Lucy 1992).

Los hablantes entran dentro de una habitación donde hay cuatro objetos colocados sobre una mesa. Uno de ellos tiene la función de distraer al sujeto (llamaré a este objeto D). Los otros tres son artilugios colocados estratégicamente en fila mirando hacia D. En la segunda parte del experimento se les pide que entren en otra habitación, donde los elementos en fila y D están posicionados en una orientación distinta, y se les pide que coloquen los objetos en la misma posición que en la que se encontraban. Los hablantes 
que normalmente utilizan el sistema absoluto ignoraron D y colocaron los objetos en la misma posición cardinal en la que se encontraban en la habitación anterior. Los hablantes que utilizan el sistema de referencia relativo colocaron los objetos tomando como referencia $\mathrm{D}$ y los hablantes que utilizan el sistema intrínseco colocaron los objetos tomando como punto de referencia la perspectiva del sujeto. Li y Gleitman $(1999 ; 2002)$ realizaron el experimento obteniendo un resultado distinto al de Levinson. El experimento ha sido llevado a cabo posteriormente varias veces con resultados diversos. El estudio de Landau et al (2010) sugiere que el LN tiene efectos transitorios en la modulación de la cognición espacial. La evidencia empírica que se extrae del debate da a entender que puede haber una relación entre los sistemas de categorización espacial y la forma en que los hablantes colocan o posicionan los objetos, pero no se ha verificado la existencia de relación causal. En caso de haberla, esta relación es probablemente transitoria en el sentido que veíamos con Casasanto (2007).

Los estudios muestran que no todas las abstracciones de relaciones espaciales son adquiridas lingüísticamente (Casasanto 2015). También sugieren que las niñas y niños prelingüísticos pueden distinguir la diferencia entre debajo de (below) y encima de (above) (Quinn et al 1996), y que reconocen la relación entre (between) (Quinn et al. 2003) antes de aprender esas palabras. Sin embargo, hay indicios de que los individuos no reconocen la relación sobre (on) antes de aprender la palabra (Casasola y Cohen 2002). Hespos y Spelke (2007) estudian si la capacidad de las niñas y niños para reconocer categorías espaciales se ve influida por el ambiente lingüístico previo a la adquisición del LN. Para ello, hacen un estudio comparativo entre sujetos que aún no conocen el idioma pero que están acostumbrados a escuchar el coreano y el inglés. Mientras que en inglés las preposiciones in y on marcan una distinción gramatical entre estar dentro de y ser soportado por, en coreano no existe esa distinción, sino una en la que los dos objetos encajan y otra en la que no. Mientras que los hablantes del inglés distinguen entre «in the carousel» («en el carrusel»), «the ring on the finger» («el anillo en el dedo») o «on the table» («en la mesa»), en coreano se habla en los dos primeros casos de «kitta» y en el tercero de «nohta». El resultado muestra que las niñas y niños son sensibles a estas diferencias incluso cuando la distinción es transversal a su ambiente lingüístico. Sin embargo, parece que los hablantes son menos sensibles a las distinciones entre las relaciones espaciales en inglés y coreano, lo que muestra que el LN influye en la capacidad de los adultos para categorizar la diferencia entre los tipos de eventos (Mc Donough, Choi y M Mandler 2003). Estos estudios sugieren que partimos de un esquema común que luego es modificado por el LN. La explicación teórica sobre el papel del LN en la cognición tiene que permitir que haya representaciones 
sobre relaciones espaciales previas al $\mathrm{LN}$ y que el $\mathrm{LN}$ pueda modificarlas dando lugar a diferencias cognitivas entre los hablantes.

\section{MECANISMOS EXPLICATIVOS DE LA INFLUENCIA DEL LN}

Hasta el momento se han aportado datos empíricos que apoyan la idea de que el LN afecta al pensamiento en determinados dominios, en concreto, a algunos pensamientos abstractos y relacionales. Previamente se propuso la hipótesis de que el LN invita de a crear categorías que sin el LN no tendríamos. Es necesario estudiar qué papel tiene el LN en la cognición y qué mecanismos son los que explican los datos. Los resultados empíricos imponen una serie de constricciones a estas explicaciones. La explicación que se proponga del fenómeno debe poder dar cuenta de los siguientes requisitos:

(i) Aunque la lengua materna tiene un papel fundamental en la formación de algunas categorías mentales, hay representaciones que son previas al LN. Esto se extrae de los experimentos sobre la adquisición de la lengua en niños en el apartado anterior (Quinn et al 1996, Quinn et al 2003, Hespos y Spelke 2003, McDonough, Choi y M Mandler 2003; Bowerman y Choi 1991; 2003).

(ii) Hay varios dominios cognitivos (como el espacial y el temporal) que están de alguna manera relacionados a través de la lengua (Casasanto 2007). Es necesario que el mecanismo explique cómo metáforas de dominios distintos (cantidad o volumen) afectan a la manera en que los hablantes piensan sobre el tiempo y puede estar relacionados con su conducta no lingüística.

(iii) Tiene que poder haber un cambio de conducta tras el proceso de habituación que se observa en el experimento de Casasanto (2007) y que consideran Landau et al (2010). La explicación no puede limitarse a la formación de estructuras fijas en la infancia, sino que el pensamiento que se ve afectado o moldeado por el $\mathrm{LN}$ tiene que poder transformarse de nuevo más adelante.

(iv) La influencia de la lengua no puede ser sólo relevante en tareas que requieran describir lo que se piensa, debe afectar a la conducta de los hablantes de la manera que muestran los experimentos de Levinson (2010) aunque solo sea de forma transitoria. Como mínimo, tiene que dar cuenta de las diferencias a la hora de estimar periodos de tiempo, según el experimento de Casasanto (2007)

(v) La influencia de la lengua debe ser mayor en ámbitos de pensamiento que involucren pensamiento abstracto y relacional. Este requisito lo imponen los experimentos de Gentner y Boroditsky (2001) al establecer que los objetos y entidades se pueden individuar prelinguisticamente -mediante la observación perceptual- del objeto. En el caso de las relaciones entre objetos o de entidades abstractas, el lenguaje tiene que jugar un papel mucho mayor. 
En adelante, presento varios mecanismos teóricos y analizo si dan cuenta de estos requisitos.

\section{IV.1. LN COMO HERRAMIENTA (LH)}

LH entiende el LN como una herramienta externa que complementa el pensamiento previo al LN (Clark 1998). Clark utiliza la metáfora del manglar para decir que el LN actúa como las raíces de un manglar. Las palabras sirven como puntos de anclaje para el pensamiento de segundo orden, creando islas de un tipo de pensamiento que es exclusivo de los seres humanos. Clark coincide con los resultados analizados en que no todo el pensamiento consiste en palabras, tesis que se le ha achacado a veces al Neowhorfismo (véase Pinker 2007, Casasanto 2007), sino que el LN participa en la formación de categorías del pensamiento de segundo orden.

Según LH, el LN es la clave por la que se redescriben nuestros propios pensamientos en un formato que los hace disponibles para otras operaciones. Afirma que una vez que tenemos el aparato lingüístico se pueden desarrollar estructuras que funcionen como andamio cognitivo para el resto del pensamiento. La explicación de Clark da a entender que el LN crea una estructura fija sobre la cual se forma el pensamiento de segundo orden.

LH no explica por qué parece que algunas representaciones del mismo nivel de pensamiento son previas al LN y otras no. Por otro lado, los puntos de anclaje del andamio lingüístico tendrían que ser lo suficientemente flexibles como para que tras un proceso de habituación al uso de formas lingüísticas distintas (de otras lenguas) pudiese haber un cambio en la conducta del individuo. De modo que LH no cumple (i) y, aunque no es incompatible con (ii), es necesario dar una explicación sobre cómo pueden cambiar los anclajes. La hipótesis de Clark no explica los resultados ofrecidos por los experimentos del neowhorfismo.

\section{IV.2. HIPÓTESIS DEL MAPEO ASOCIATIVO (MA)}

Según MA (Casasanto 2008; Casasanto y Bottini 2010; Dolscheid et al. 2012), las representaciones mentales (RM) y las relaciones intermodulares son previas al LN. Estas representaciones se adquieren gracias a la interacción con el medio físico-cultural, mediante un proceso de mapeo asociativo. El aprendizaje lleva a asociar representaciones entre módulos distintos como en el caso de la colocación espacial y el tiempo, lo que explica (ii). La asociación tiene lugar a través de la observación experiencial de fenómenos. Estos fenómenos mapean asociaciones no fijadas entre representaciones de los dos módulos. El LN lleva a cabo un proceso de fijación de las representaciones y de las asociaciones existentes, que aporta una mayor tendencia al uso de esas 
representaciones o asociaciones en tareas no lingüísticas.

La fijación de algunas representaciones y asociaciones para que sean más comunes no tiene que ser permanente, el uso de nuevas formas lingüísticas puede hacer que se fijen nuevas asociaciones o representaciones, cambiando la conducta de los individuos, lo que da cuenta de (iii). MA explica la variación de la conducta de los hablantes del griego y del inglés al estimar la duración de los estímulos. El uso de metáforas espaciales y de cantidad fijaría unas determinadas asociaciones entre las representaciones de distintos módulos (espacio-tiempo y cantidad-tiempo) que hacen que la estimación del tiempo sea distinta para los hablantes de una y otra lengua. Tras la habituación al uso de metáforas de otro tipo, la fijación de las asociaciones cambia, haciendo que cambie también la estimación temporal, como exige (iv).

El mecanismo no explica que haya estudios que muestren que algunas categorías de colocación espacial no son adquiridas hasta que no se aprende la lengua materna, como se ha visto con (i). Según MA, estas representaciones tienen que ser previas al LN. El LN fija las representaciones una vez que ya son adquiridas.

Aunque MA cumple (ii), (iii) y (iv), no cumple (i), ya que no es suficiente que las categorías se fijen con la adquisición de la lengua. Algunas categorías tienen que conformarse a partir de la adquisición del LN, no pudiendo darse previamente.

\section{IV.3. HIPÓTESIS DE LOS CONCEPTOS LÉXICOS Y MODELOS COGNITIVOS (LCCM)}

La LCCM (Evans 2009) considera que aunque los sistemas de conceptos léxicos y conceptos cognitivos son diferentes, hay una interacción entre el sistema conceptual y el lingüístico integrada en modelos cognitivos previos al lenguaje. Evans (2009) reconoce que la información proposicional acumulada a través de rutas lingüísticas puede ser incorporada en los modelos cognitivos. Durante el desarrollo cognitivo, la interacción entre los dos sistemas puede modificar los estados representacionales del sistema conceptual, proveyendo un recurso de información adicional. El lenguaje funcionaría como un indicador que, debido a las diferencias interlingüísticas, da lugar a diferencias cognitivas entre los hablantes de distintas lenguas. Evans (2012) afirma que los usuarios de cualquier lengua deben prestar atención a distintos aspectos de la realidad para poder empaquetarlos según sus necesidades comunicativas. Ese proceso de empaquetamiento tiene consecuencias profundas en la cognición no lingüística, haciendo que la lengua influya en cómo categorizamos aspectos de nuestro medio socio-físico.

Para que LCCM sea compatible con (i), habría que suponer que los conceptos no lingüísticos se dan en la cognición como aspectos de la realidad 
no empaquetados antes de la adquisición del LN. Puesto que hay una relación entre el LN y los conceptos no lingüísticos, durante el aprendizaje de estos conceptos léxicos, el LN funcionaría como indicador de qué conceptos cognitivos deben agruparse según las necesidades comunicativas de la lengua. El uso de la palabra indicaría la necesidad cognitiva de empaquetar la información del modelo cognitivo en una misma categoría.

En el caso del estudio de las metáforas verticales y horizontales del chino mandarín de Boroditsky (2001), las metáforas funcionarían como indicador de qué tipo de conceptos se pueden empaquetar juntos. En el caso del inglés se agruparían los conceptos de direccionalidad horizontal con los de sucesión temporal y en el caso del chino mandarían los de direccionalidad vertical con los de sucesión temporal. Ello causaría las diferencias interlingüísticas tanto a la hora de describir la realidad como al realizar tareas de tipo no lingüístico. Esto explicaría (i), pero Evans no elabora una explicación. Para que la tesis fuese satisfactoria habría que desarrollar la hipotesis en detalle.

La tesis de Evans (2003) es que la relación entre el sistema léxico y el cognitivo se reduce a los conceptos léxicos de clase abierta -que en inglés refieren a verbos, sustantivos, adverbios y adjetivos- pero no sirve para explicar conceptos relacionales y preposiciones. Esto sirve para explicar por qué las niñas y niños dan muestras de reconocer algunas representaciones de relaciones espaciales (Quinn et al. 1996; Quinn et al. 2003) y sin embargo parece que hay diferencias interlingüísticas en la categorización de sucesos como abrir (Bowerman y Choi 2003). LCCN tiene problemas para dar cuenta de los estudios que afirman que los individuos no reconocen la relación sobre (on) antes de aprender la palabra (Casasola y Cohen 2002), ni explican que las diferencias del desarrollo cognitivo de quienes hablan lenguas muy diferentes son mayores en el aprendizaje de palabras que designan relaciones que en las que designan entidades y propiedades (sustantivos y adjetivos) (Gentner y Boroditsky 2001). LCCM tiene problemas con (v), con lo que no puede ser la explicación que buscamos.

\section{IV.4. HiPÓTESIS DE LA CALIFICACIÓN O ETIQUETAJE (EL)}

Según EL, nombrar un objeto o suceso determinado ayuda al niño o niña a individualizar los objetos o los sucesos que componen un evento. Algunos estudios muestran que la presencia de etiquetas de este tipo (por ejemplo, decir «imira, un pato!» mientras se presenta un estímulo en el que hay un pato) ayuda a individuar el objeto. Una etiqueta común motiva a atender rasgos comunes de su ambiente y esto ayuda a la formación de una categoría (Boroditsky y Gentner 2001; Gentner y Namy 1999). Los estudios de Casasola (2005) apuntan a que oír la palabra «on» ayuda a las niñas y niños a formar la categoría espacial sobre. El mecanismo por el cual sucede esto ha sido 
explicado de diversas formas. Xu (2002) ofrece varias hipótesis:

La primera es que el LN ayuda en la capacidad limitada de la memoria durante el procesamiento de información. Cuando la tarea que tienen que hacer las niñas y niños es sencilla, son capaces de usar la información de los rasgos del objeto para individuarlo bajo circunstancias determinadas. Si la tarea es demasiado complicada, la presencia de etiquetas ayuda a seguir la representación de los objetos en mente y retenerlos después para tener éxito en la tarea. Esta propuesta no explica que haya diferencias en la realización de tareas no lingüísticas entre hablantes de lenguas distintas. El papel del LN es meramente ayudar a memorizar una serie de procesos durante el desarrollo cognitivo temprano, pero no tiene ninguna repercusión en la cognición adulta. De modo que no puede ser la explicación que buscamos.

La segunda hipótesis es que distintas etiquetas destacan diferencias entre rasgos y propiedades de distintos objetos. Una vez que la niña o niño es consciente de esas diferencias, es más probable que concluya que los objetos que se etiquetan con nombres distintos son dos objetos diferentes. Según esta explicación, el LN influye en las representaciones de los objetos porque la presencia de etiquetas atrae la atención de la niña o niño hacia determinados rasgos del objeto y eso le hace llegar a conclusiones acerca del número de objetos presentes en un suceso.

Una tercera versión es que las palabras funcionan como marcadores de esencias. Las palabras mapean distintas clases o tipos en su ambiente. Oír o memorizar una palabra no es suficiente para que se forme el concepto completo, pero la palabra puede dirigir el proceso al configurar marcadores que indican cuáles son los conceptos relevantes. La interacción con el mundo hace que los conceptos sean elaborados posteriormente.

Para que estas versiones expliquen las diferencias en el dominio espacial relacional, el etiquetaje no puede influir sólo en la apreciación de rasgos de objetos e individuación de esencias, tiene que afectar a la formación de categorías relacionales como encima de. El niño tendría que ser capaz de observar las diferencias entre objetos que están encima de otros y objetos que se encuentran en otra posición cuando se le dice: «mira, la pelota está encima de la mesa»o «el teléfono está sobre la silla». Es posible que la palabra o la expresión «encima de» funcione como marcador para observar las diferencias y las semejanzas entre estos eventos y que eso ayude a la formación del concepto. En tal caso, la palabra no sirve como indicador para individuar sólo objetos o esencias, sino también relaciones. Estas versiones de la teoría no son suficientes para dar cuenta de los estudios. Para poder explicar (v) tendrían que ir más allá de los objetos y explicar cómo la lengua influye en la formación de categorías relacionales.

Una versión más fuerte de la hipótesis del etiquetaje es la que afirma que 
el LN influye en la arquitectura conceptual (Xu 2002). Aprender el nombre de las cosas o de eventos haría que los individuos relacionen diferentes aspectos sobre la representación de un objeto. Los hablantes tendrían dos rutas para codificar los rasgos característicos del objeto. Durante la infancia temprana estas vías están muy separadas. Al aprender las palabras se tienen que satisfacer dos tipos constricciones: las constricciones taxonómicas de la palabra y las constricciones del objeto. Estas constricciones hacen que la niña o niño preste atención a la información de localización y de rasgos del objeto durante la formación de la categoría. Esta versión es compatible con los estudios de Casasola (2005) que muestran que el etiquetaje de «on» ayuda a la formación de la categoría mental de estar sobre. Las constricciones taxonómicas de la palabra «on» y las constricciones dadas por la relación ente los dos objetos harían que se preste atención a dos tipos de información: tanto a los rasgos de los objetos, como a la localización que tienen, formándose así la categoría mental que le permite individuar los objetos y las relaciones espaciales entre ellos.

Siguiendo EL, las diferencias léxicas entre las distintas lenguas llevarán a diferencias cognitivas porque hay diferencias en qué palabras se aplican a unos eventos y a otros. Esto explica las diferencias que se observan entre las niñas y niños con el coreano y el inglés (Bowerman y Choi 1991; 2003). Al usar una etiqueta distinta para hablar de unos eventos u otros en una lengua y en otra la misma hace que las niñas y niños se fijen en rasgos distintos del evento, codificándose la palabra de forma diferente.

La hipótesis sigue estando en tensión con (v), si la influencia del LN consiste en que las palabras nos hacen relacionar rasgos de objetos, entonces, las diferencias interlingüísticas no deberían ser mayores en ámbitos relacionales y abstractos. Aunque puede explicarse afirmándose que las etiquetas tienen un efecto mayor al nombrar eventos que involucran varios objetos o acciones que al hablar de objetos materiales concretos, el fenómeno debería ser explicado.

Por otro lado, para que EL explique los resultados de Casasanto (2007) y Landau (2010) los conceptos de individuación de eventos y objetos tienen que ser flexibles y cambiantes. Tras un proceso de habituación al uso de metáforas espaciales para hablar del tiempo, el hablante del griego sufriría un cambio en su arquitectura conceptual que dé lugar a una variación en su conducta. Sólo en este caso EL podría dar cuenta de (iii). Aunque esta explicación puede hacerse compatible con los cinco requisitos, tiene varias tensiones que habría que solventar.

\section{IV.5. TEORÍA DE LA REDESCRIPCIÓN DE REPRESENTACIONES (RR)}

Según RR, una vez se han formado las RM prelingüísticas, puede haber un proceso de grabación posterior que se vea influido por el LN (Karmiloff-Smith 
1992). Las diferencias interlingüísticas en cómo se organizan semánticamente los datos perceptivos pueden afectar a los estados iniciales de la formación de representaciones debido a un proceso de redescripción que tendría lugar durante el aprendizaje de la lengua (Majid et al 2004). Según RR, hay tres tipos de recursos complementarios en la formación de categorías: predisposiciones innatas, adquisición de datos a través de la interacción con el ambiente físico y sociocultural y procesos internos de cambio representacional en los que la mente se enriquece a sí misma dentro del conocimiento re-representacional. El primer nivel de conocimiento explica que haya indicios de que se tienen representaciones sobre algunas relaciones espaciales como muestran los estudios de Quinn et al. (1996); Quinn et al. (2003). Durante el segundo estado del aprendizaje - esto es, la adquisición de datos del entorno- el conocimiento sigue sin estar disponible al acceso consciente o a la descripción verbal. Estos procesos sólo se hacen disponibles tras el proceso de redescripción que permite además, que haya una conciencia metalingüística, en la que el niño trata al lenguaje como un objeto. (Karmiloff-Smith y Clark 1993).

La influencia del LN tiene lugar durante la redescripción de representaciones. Antes de la adquisición de la lengua, el conocimiento es representado y activado en respuesta a un estímulo externo. Con el LN y la interacción con el entorno físico, se da un proceso de redescripción que crea estructuras nuevas de información. Las estructuras lingüísticas permiten el desarrollo de nuevas estructuras conceptuales. Las variaciones léxicas interlingüísticas suponen, entonces, diferencias en esas formas de guía de la redescripción de representaciones, dando lugar a variaciones en la estructura conceptual de algunos dominios cognitivos. Las variaciones en el proceso de redescripción darían lugar a las diferencias en la conducta no lingüística que se observa en los estudios.

Para que el fenómeno no sea permanente (Casasanto 2007), la información re-representacional tiene que ser maleable y flexible, de modo que con el uso de nuevas formas lingüísticas, la información pueda redescribirse alterando la conducta no lingüística de los hablantes. Sólo entonces la teoría podría dar cuenta de (iii). Siendo esto así, RR explica todos los requisitos impuestos.

En primer lugar, el desarrollo del aprendizaje en tres fases explica que haya representaciones previas a la lengua y también que la lengua influya en la formación de nuevas estructuras de pensamiento que determinan las diferencias interlingüísticas en algunos dominios, tal y como exige (i). Al crearse nuevas estructuras durante la redescripción, a través del lenguaje, algunas estructuras pueden relacionarse con otras, de modo que al utilizarse metáforas de otros dominios para hablar del tiempo -como en el caso de las metáforas espaciales o de cantidad-, estas metáforas redescriben representaciones previas, creando estructuras nuevas de información conceptual que relacionan ambos ámbitos 
de pensamiento e influyen en la conducta de los hablantes. Esto explica (ii). La redescripción de representaciones no sólo afecta al dominio lingüístico, sino que crea nuevas estructuras de información sobre las representaciones anteriores, lo que explica que haya variaciones en la conducta no lingüística además de en la lingüística como exige (iv). La segunda fase explica que la lengua pueda influir más en el pensamiento relacional o abstracto ya que existe un estadio previo al de re-representación en el que el niño o niña aprende a través de la percepción e interacción con el medio. Esto encaja con que la individuación de los objetos físicos a través de sus rasgos sea anterior a la individuación de relaciones, haciendo que el papel de LN sea mayor en el pensamiento relacional. Esta idea cumple con (v).

RR puede dar cuenta de todos los requisitos que se han extraído de los experimentos. Esto no significa que RR sea la única explicación válida. Puede haber muchas otras explicaciones que den cuenta de los estudios y que no se hayan considerado aquí. Además, algunas de las teorías expuestas, aunque presentan tensiones con los requisitos que se imponen, pueden solventar sus dificultades desarrollando ciertos puntos de la explicación teórica. La RR es, sin embargo, la que mejor da cuenta de todas las exigencias que imponen los estudios empíricos.

\section{CONCLUSIONES}

A lo largo del trabajo se han analizado estudios empíricos sobre la influencia de la lengua en el pensamiento de dominio racional y abstracto. Se han extraído cinco requisitos que cualquier explicación acerca de cómo la lengua influye en el pensamiento debería cumplir si se desea que el mecanismo de cuenta de los resultados empíricos. Por último, se han analizado cinco teorías sobre la influencia de la lengua en el pensamiento y se ha estudiado si cumplen o no estos requisitos. Finalmente, se ha argumentado que la hipótesis que mejor da cuenta de los requisitos extraídos es la tesis de la re-representación.

\section{REFERENCIAS BIBLIOGRÁFICAS}

ACERO, J. J. 2013: «Las variedades de la relatividad lingüística: De Whorf al Neowhorfismo», en J. F. García Casanova y A. Vallejo, eds. Crítica y meditación. Homenaje al profesor Pedro Cerezo Galán. Granada: EUG.

BORODITSKY, L. 2001: «Does language shape thought? Mandarin and English Speakers Conception of time», Cognitive psychology 43:1, 1-22.

BORODITSKY, L. y GENTNER, D. 2001: «Individuation, relativity, and early word learning», en Bowerman, M., y Levinson, C. (eds.):. Language cognition and conceptual development, Cambridge: Cambridge University Press. 
BORODITSKY, L. y PRINZ, J. 2008: «What thoughts are made of en Semin», en G.R.; Smith, E. R. (eds.): Embodied Grounding: Social, Cognitive, Affective, and Neuroscientific Approaches. Cambridge: Cambridge University Press.

BOWERMAN, M. y CHOI, S. 1991: «Learning to express motion events in English and Korean: The influence of language-specific lexicalization patterns», Cognition, 41(1): 83-121.

BOWERMAN, M., y CHOI, S. 2003: «Space under construction: Language-specific spatial categorization in first language acquisition», en Gentner, D. y GoldinMeadow, S. (eds.): Language in mind: Advances in the study of language and cognition, Cambridge, M.A: The MIT Press, pp.387-428.

BROWN, P. y LEVINSON, S. C. 2000: «Frames of spatial reference and their acquisition in Tenejapan Tzeltal», en Nucci, L., Saxe, G. B., y Turiel, E. (eds): Culture, thought, and development, New Yersey London: Psychology Press, pp. 167-197.

CAREY, S. 2009: The origin of concepts. New York: Oxford University Press.

CASASANTO, D. 2005: «Crying 'Whorf’!», Science 307: 1721-1722.

CASASANTO, D. 2008: "Who's afraid of the big bad Whorf? Crosslinguistic differences in temporal language and thought», Language learning 58: 1, 63-79.

CASASANTO, D. 2014: «Development of metaphorical thinking: the role of language», en M. Borken, M., Hunnell, J. y Dancygier,B. (eds.): Language and creative mind. Stanford, CA: CSLI Publications.

CASASANTO, D. y BORODITSKY, L. 2007: «Time in the mind: Using space to think about time», Cognition, 106(2): 579-593.

CASASANTO, D. y BOTTINI. R 2010: "Can mirror-reading reverse the flow of time?», en Hölscher, C., Shipley, T.F., Olivetti Belardinelli, Bateman, J. A., Newcombe, N. S.: Spatial cognition VII, Berlin Heidelberg: Springer, pp. 33545 .

CASASOLA, M. 2005: «Can language do the driving? The effect of linguistic input on infants' categorization of support spatial relations», Developmental psychology 41: 183-92.

CASASOLA, M. y COHEN, L. B. 2002: «Infant categorization of containment, support and tight-fit spatial relationships», Developmental science, 5(2): 247 264.

CLARK, A. (1998): «Magic words: How language augments human computation», en Carruthers, P. y Boucher, J. (eds.): Language and thought: Interdisciplinary themes, Cambridge: Cambridge University Press, pp. 162-183.

DOLSCHEID, S., HUNNIUS, S., CASASANTO, D., y MAJID, A. 2012: «The sound of thickness: Prelinguistic infants' associations of space and pitch», en The 34th annual neeting of the cognitive science society. Cognitive Science Society, pp. 306-311.

GELMAN, R. y GALLISTEL, C.R, 2004: «Language and the origin of numerical 
concepts», Science 306: 441-443.

GENTNER, D. y NAMY, L. L. 1999: «Comparison in the development of categories» Cognitive development 14(4): 487-513.

GORDON, P. 2004: «Numerical cognition without words: Evidence from Amazonia», Science 306 (5695): 496-499.

EVANS, V. 2009: How words mean: lexical concepts, cognitive models and meaning construction, Oxford: Oxford University Press.

EVERETT, C. 2013: Linguistic relativity. Evidence across languages and cognitive domains, Berlin: De Gruyter Mouton.

HESPOS, S. J. \& SPELKE, E. S. 2007: «Precursors to spatial language: The case of containment», en: Aurnague, M., Hickmann, M., \& Vieu, L. (eds.): The categorization of spatial entities in language and cognition (Vol. 20). (pp. 233245) Amsterdam/Philadelphia: John Benjamins Publishing.

JANUARY, D. y KAKO, E. 2007: «Re-evaluating evidence for linguistic relativity: Reply to Boroditsky 2001». Cognition 104(2): 417-426.

KARMILOFF-SMITH, A. 1992: Beyond modularity: a developmental perspective on cognitive science. Cambridge, MA: MIT Press.

KARMILOFF-SMITH y CLARK,A. 1993: «The cognizer's innards: A psychological and philosophical perspective of the development thought», Mind and language 8(4): 487-519.

LANDAU, B., DESSALEGN, B. y GOLDBERG, A. M. 2010: «Language and space: Momentary interactions», en Chilton, P. y Evans, V. (eds): Language, cognition, and space: The state of the art and new directions, London: Equinox, pp.51-78.

LEVINSON, S. C. 2003: Space in language and cognition, Cambridge: Cambridge University Press.

LI, P. y GLEITMAN, L 1999: Language and spatial reasoning, MS. University of Pennsylvania.

LI, P. y GLEITMAN, L 2002: «Turning the tables: Language and spatial reasoning», Cognition 83(3): 265-294.

LI, P., DUNHAM, Y. y CAREY, S., 2009: «Of substance: The nature of language effects on entity construal. Cognitive Psychology 58: 487-524.

LUCY, J. 1992: Language diversity and thought. New York: Cambridge University Press.

MAJID, A., BOWERMAN, M., KITA, S., HAUN, D. B., y LEVINSON, S. C. 2004: «Can language restructure cognition? The case for space. Trends in cognitive sciences, 8(3): 108-114.

MCDONOUGH, L., CHOI, S. y MANDLER, J. M. 2003. «Understanding spatial relations: Flexible infants, lexical adults», Cognitive psychology, 46(3): 229-259.

MILLER, K., MAJOR, S. M., SHU, H. y ZHANG, H. 2000: «Ordinal knowledge: 
Number names and number concepts in Chinese and English», Canadianjournal of experimental psychology/revue canadienne de psychologie expérimentale 54(2): 129.

NÚÑEZ, R. E. y SWEETSER, E. 2006: «With the future behind them: Convergent evidence from Aymara language and gesture in the crosslinguistic comparison of spatial construals of time», Cognitive science, 30(3), 401-450.

PICA, P., LEMER, C., IZARD, V. y DEHAENE, S. 2004: «Exact and approximate arithmetic in an Amazonian indigene group», Science 306(5695): 499-503.

PEDERSON, E. 1995: «Language as context, language as means: Spatial cognition and habitual language use», Cognitive Linguistics 6: 33-62.

PINKER, S. 2007: The stuff of thought, New York: VIKING

PRINZ, J. y REINES, M. F. 2009: «Reviving Whorf: The return of linguistic relativity», Philosophy Compass 4(6):1022-1032

QUINN, P. C., CUMMINS, M., KASE, J., MARTIN, E. y WEISSMAN, S. 1996: «Development of categorical representations for above and below spatial relations in 3-to 7-month-old infants», Developmental Psychology 32(5): 942950.

QUINN, P. C., ADAMS, A., KENNEDY, E., SHETTLER, L. y WASNIK, A. 2003: «Development of an abstract category representation for the spatial relation between in 6-to 10-month-old infants», Developmental Psychology 39(1): 151163.

SAPIR, E 1929: «The Status of Linguistics as a Science\| en Language», Linguistic society of America 5:4, 207-214.

SPELKE, E. S., y TSIVKIN, S. 2001: «Language and number: A bilingual training study», Cognition 78(1), 45-88.

VICENTE, A. y MARTÍNEZ-MANRIQUE, F. 2013: «The influence of language in conceptualization: three views», Protosociology 20: 89-106

XU, F. 2002: «The role of language in acquiring kind concepts in infancy», Cognition 85: $223-50$.

WHORF, B. L. 1956/2012: Language, thought and reality: selected writings of Benjamin Lee Whorf. Ed. Carroll, J. B., Cambridge, Masachusetts: The MIT Press.

MARINA ORTEgA-ANDRÉS ES Investigadora predoctoral FPI 2015, Departamento de Lingüística y Estudios Vascos, UPV/EHU.

Líneas de Investigación:

Filosofía del lenguaje, semántica léxica, pragmática.

Publicaciones recientes: 
(2016): «Polisemia y conceptos», en Actas de congresos, VIII Congreso de la Sociedad Española de Filosofía Analítica, SEFA 2016, pp. 137-139. Disponible en http:// sefaweb.es/sefa2016/sefa_congreso_oviedo_2016\%20Abstract.pdf

(2016): «Polisemia e infraespecificación semántica», Actas de congreso: III Congreso de Graduados, Sociedad de Lógica, Metodología y Filosofía de la ciencia) SSN: 1577-2292 SLMFCE, pp. 40-43. Disponible en http://www.solofici.org/system/ files/documentos/iii_conferencia_graduados_fin.pdf

Correo electrónico: marina.ortega@ehu.eus 